\title{
Procedimiento de Puestow: resultados en 19 años de experiencia institucional
}

\author{
Puestow procedure: results in 19 years of institutional experience
}

\author{
José Roberto Contreras-Ramírez, ${ }^{*}$ Ismael Domínguez-Rosado, ${ }^{\ddagger}$ \\ Luis Carlos Chan-Núñez, ${ }^{\S}$ Paulina Carpinteyro-Espin, \\ Estefanía Carrillo-Navarrete," Manuel Campuzano-Fernández ${ }^{* *}$
}

Palabras clave: Pancreatitis crónica, pancreatoyeyunoanastomosis lateral, procedimiento de Puestow.

Keywords: Chronic pancreatitis, lateral pancreaticojejunostomy, Puestow procedure.

* Fellow de Cirugía Hepatopancreatobiliar, México.

‡Cirujano

Hepatopancreatobiliar, Médico adscrito al Departamento de Cirugía General, México.

$\S$ Coordinador de la Clínica de Hepatopancreatobiliar, Médico adscrito al Departamento de Cirugía General, México. "Cirujano general, Departamento de Trasplantes, México. " Médico Pasante en Servicio Social, México. ** Departamento de Cirugía General y Clínica de Páncreas, México.

Instituto Nacional de Ciencias Médicas y Nutrición "Salvador Zubirán”.

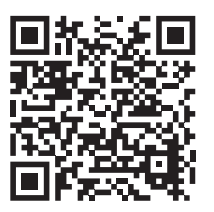

\section{RESUMEN}

Introducción: La pancreatitis crónica comprende un síndrome inflamatorio progresivo crónico del páncreas, con daño irreversible y pérdida de la función exocrina y endocrina. El dolor es el síntoma más frecuente. El tratamiento quirúrgico es superior en comparación con terapias conservadoras o endoscópicas. Objetivo: Conocer los resultados obtenidos en la realización de un procedimiento de Puestow para el manejo de pancreatitis crónica. Material y métodos: Se incluyeron todos los pacientes que fueron sometidos a un procedimiento de Puestow, en el Instituto Nacional de Ciencias Médicas y Nutrición “Salvador Zubirán” entre los años 2000 a 2019. Resultados: Se encontraron 45 pacientes operados por una pancreatoyeyunoanastomosis lateral. La edad media fue de 46 años. Fueron 24 (53\%) hombres y 21 (47\%) mujeres. En 42.2\% de los pacientes había antecedente de alcoholismo y/o tabaquismo. Las indicaciones más frecuentes para la cirugía fueron: dolor (46.7\%) y pancreatitis aguda a repetición (37.8\%). Dieciocho (40\%) pacientes cursaron con recurrencia del dolor con un seguimiento medio de 4.4 años. Conclusiones: En la actualidad, no existe un procedimiento quirúrgico ideal para pacientes con pancreatitis crónica, el tipo de intervención a elegir debe ser el reflejo del equilibrio entre el éxito del procedimiento y los riesgos inherentes.
Introduction: chronic pancreatitis includes a chronic progressive inflammatory syndrome of the pancreas, with irreversible damage and loss of exocrine and endocrine function. Pain is the most frequent symptom. Surgical treatment is superior in comparison to conservative or endoscopic therapies. Objective: Learn the results obtained when performing a Puestow procedure for the management of chronic pancreatitis. Material and methods: All patients who underwent a Puestow Procedure in the National Institute of Medical Sciences and Nutrition "Salvador Zubirán" between 2000 and 2019, were included. Results: 45 patients with a lateral pancreaticojejunostomy were found. The mean age was 46 years. There were 24 (53\%) men and 21 (47\%) women. Alcoholism and/or smoking history was found in $42.2 \%$ of the patients. The most frequent indications for surgery were: pain (46.7\%) and acute recurrent pancreatitis (37.8\%). Eighteen patients (40\%) had recurrence of pain with a mean follow-up of 4.4 years. Conclusions: At the time, there is no ideal surgical procedure for patients with chronic pancreatitis, the type of intervention to be chosen should reflect a balance between the success of the procedure and the inherent risks.

\section{INTRODUCCIÓN}

L a pancreatitis crónica (PC) comprende un conjunto de manifestaciones clínicas secundarias a un proceso inflamatorio persistente del páncreas. ${ }^{1-3}$ La Asociación Americana del
Páncreas (AAP) la define como: "un síndrome inflamatorio y de cicatrización progresiva crónica del páncreas, con daño irreversible y pérdida de la función exocrina y endocrina". ${ }^{4}$

La PC muestra una incidencia global anual de cuatro a 23 casos por 100,000 habitantes,

Citar como: Contreras-Ramírez JR, Domínguez-Rosado I, Chan-Núñez LC, Carpinteyro-Espin P, Carrillo-Navarrete E, Campuzano-Fernández M. Procedimiento de Puestow: resultados en 19 años de experiencia institucional. Cir Gen. 2021; 43 (1): 15-22. https://dx.doi.org/10.35366/103909 
Recibido: 22/06/2020 Aceptado: 30/07/2021 y una prevalencia de 13 por $100,000 .{ }^{5}$ Los pacientes tienen un riesgo de mortalidad cuatro veces mayor en comparación con la población general, y diez veces más probabilidad de malignidad pancreática. ${ }^{6}$ Son múltiples las etiologías que se atribuyen al desarrollo de PC. En Estados Unidos el consumo de alcohol es responsable de $45 \%$ de los casos reportados. Otras causas se incluyen en el acrónimo TIGAR-O (Toxic) metabolic, idiopathic, genetic, autoimmune, recurrent severe acute pancreatitis, obstructive). ${ }^{7}$

Una hipótesis ligada a la pancreatitis hereditaria sugiere que la PC empieza con un episodio de pancreatitis aguda, este modelo se conoce como SAPE (Sentinel Acute Pancreatitis Event). ${ }^{8}$ El dolor abdominal es el síntoma más frecuente de la PC. Se han propuesto diferentes mecanismos causales del dolor: por inflamación, por aumento de la presión dentro del conducto o del parénquima, por estenosis y/u obstrucción ductal por cálculos, por complicaciones como pseudoquistes o flemones, o extrapancreáticas como trombosis portal, estenosis biliar o duodenal y úlceras pépticas. ${ }^{9}$ Se ha demostrado que existen también mecanismos de sensibilización central y periférica que ocasionan cambios a nivel de la inervación pancreática. ${ }^{10}$

La Organización Mundial de la Salud (OMS) recomienda, para el tratamiento del dolor, iniciar con medicamentos no opioides, y si no hay mejora pasar a opioides débiles como el tramadol seguido de opioides más potentes. ${ }^{11}$ Algunos grupos sugieren que el tratamiento endoscópico o de intervención es el primer paso para el tratamiento de pacientes con PC; sin embargo, no todos son candidatos a este tipo de abordaje. ${ }^{12,13}$

De manera reciente, se ha visto que el abordaje quirúrgico muestra superioridad para el tratamiento de PC en comparación con terapias conservadoras o endoscópicas. ${ }^{14-16}$

El procedimiento de Puestow fue descrito por primera vez en 1958 por Partington y Rochelle, se trata de un procedimiento de drenaje realizando una pancreatoyeyunoanastomosis latero-lateral longitudinal. Este tipo de cirugía es la de elección cuando se encuentra dilatado el conducto pancreático principal (diámetro mínimo de $5 \mathrm{~mm}),{ }^{17}$ en ausencia de una masa inflamatoria en la cabeza del páncreas o de obstrucción del conducto biliar. ${ }^{18}$
Se ha descrito como técnicamente seguro y eficiente, con bajas tasas de morbilidad $(20 \%)$ y mortalidad operatoria $(1 \%) .{ }^{19}$ Las fugas pancreáticas se desarrollan en menos de $5 \%$ de los casos. ${ }^{20}$ La función endocrina y exocrina usualmente no se ve comprometida, esto debido a que no hay mayor resección de tejido pancreático. En México, González y colaboradores describieron en 1996 los resultados obtenidos en 49 pacientes sometidos a una pancreatoyeyunoanastomosis por PC, y con seguimiento medio de 6.5 años, encontraron que el $98 \%$ no tenía dolor. ${ }^{21}$

Dite y colegas demostraron en el primer ensayo controlado aleatorizado, que existe un mejor control del dolor a cinco años en los pacientes que se sometieron a cirugía (34$52 \%)$, en comparación con los que recibieron tratamiento endoscópico (15-46\%). ${ }^{22}$ En la actualidad, no existe consistencia en las diferentes guías respecto al momento y elección del tratamiento de la PC.

El objetivo principal de este estudio es conocer los resultados obtenidos en la realización de un procedimiento de Puestow para el manejo quirúrgico de PC, en 19 años de experiencia en un centro de alto volumen de la Ciudad de México.

\section{MATERIAL Y MÉTODOS}

Se incluyeron todos los pacientes que fueron sometidos a un procedimiento quirúrgico tipo Puestow para el tratamiento de PC, en el Instituto Nacional de Ciencias Médicas y Nutrición "Salvador Zubirán" entre los años 2000 a 2019. Para la selección de sujetos se solicitó al Departamento de Estadística un listado con los pacientes que hubieran sido operados por derivación del conducto pancreático a intestino delgado con abordaje abierto o laparoscópico, según el código CIE-10 de procedimientos.

Se realizó una revisión de los expedientes físicos y en electrónico, recolectando los datos filtrados que cumplieron con los criterios de inclusión en una base de datos electrónica, la cual incluyó las variables propuestas. Se realizó un análisis estadístico descriptivo y comparativo dependiendo del tipo de variable. Para las variables continuas de edad, peso, índice de masa corporal (IMC), índice tabáquico, episo- 
dios de pancreatitis aguda (PA), diámetro del conducto pancreático y seguimiento en años de los pacientes operados, se calculó la media, mediana, desviación estándar e intervalos con una confianza del 95\%.

Se obtuvieron valores de frecuencias y porcentajes para las variables categóricas como sexo masculino, diabetes mellitus (DM) tipo 2, consumo de alcohol, tabaquismo, PA, insuficiencia pancreática endocrina y exocrina, estudios de gabinete como ultrasonido transendoscópico (USTE), resonancia magnética (RM) y tomografía axial computarizada (TAC). Otras variables categóricas incluyeron los hallazgos preoperatorios y transoperatorios como calcificaciones pancreáticas, presencia de litos, atrofia pancreática, así como intervenciones previas como endoscopia, drenaje de pseudoquiste pancreático, necrosectomía y uso de enzimas pancreáticas.

Se analizaron variables relacionadas a la evolución postoperatoria, tales como el uso de drenajes, desarrollo de fístulas pancreáticas, días de hospitalización y complicaciones según la escala de ACCORDION, y variables de seguimiento como la recurrencia de dolor, uso de opioides, desarrollo de insuficiencia pancreática de novo, reingresos hospitalarios y seguimiento en años. En el análisis bivariado $U$ Mann-Whitney se utilizó el programa IBM SPSS Statistics v24. Un valor de p menor a 0.05 se consideró estadísticamente significativo.

Se excluyeron del estudio todos aquellos pacientes que hubieran sido operados por el procedimiento de Puestow por una causa diferente a pancreatitis crónica, así como los que fueron operados en combinación con cualquier otro tipo de procedimiento quirúrgico de resección pancreática. Se eliminaron de la base de datos los pacientes que no fueron operados en el Instituto Nacional de Ciencias Médicas y Nutrición "Salvador Zubirán", y que no contaban con un expediente físico o electrónico completo para la recolección de datos.

\section{RESULTADOS}

Se encontraron 45 pacientes operados por un procedimiento de Puestow, secundario al diagnóstico de PC. La edad media fue de 46 años. Fueron 24 (53\%) hombres, 21 (47\%) mujeres, y el índice de masa corporal promedio fue de
$22.71 \mathrm{~kg} / \mathrm{m}^{2}$. De los pacientes con PC, cinco tenían diagnóstico de diabetes tipo 2 (11.1\%). En cuanto a episodios de pancreatitis aguda previo a la cirugía, 37 (82.2\%) habían presentado por lo menos un episodio, y la media general de estos fue de 5.2 episodios (Tabla 1).

El 42.2\% de los pacientes (19 de 45) tenían historia de consumo de alcohol o antecedentes de alcoholismo, y $42.2 \%$ tenían antecedente de tabaquismo, con una media de índice tabáquico de 22.7 (Tabla 1). En cuanto a procedimientos endoscópicos previos a la cirugía, al menos 15 pacientes (33.3\%) fueron sometidos a algún tipo de intervención para el tratamiento de PC. Se detectaron 14 casos (31.1\%) con insuficiencia endocrina, caracterizada por diabetes tipo 2 posterior al diagnóstico de PC, mientras que 19 pacientes (40\%) refirieron presentar diarrea; sin embargo, sólo nueve (20\%) tenían diagnóstico de insuficiencia exocrina.

En los estudios de gabinete se encontraron como hallazgos patognomónicos de PC a 32 (71.1\%) pacientes con calcificaciones, la mayor parte localizadas en la cabeza del páncreas $(\mathrm{n}=$ 16.50\%), 12 (37.5\%) de localización múltiple y el resto presentaron una distribución modal en el cuello $(n=1,3.1 \%)$, cuerpo $(n=2,6.2 \%)$ y proceso uncinado $(n=1,3.1 \%)$, respectivamente. La media del diámetro del conducto reportada fue de $8.03 \mathrm{~mm}$ (Tabla 1$)$.

En 15 (33.3\%) pacientes se reportó la presencia de litos, localizados en la cabeza $(\mathrm{n}=$ $11,73 \%)$, cuello $(n=1,7 \%)$ y en múltiples partes del páncreas $(\mathrm{n}=3,20 \%)$. Catorce (31\%) de los sujetos presentaron atrofia de la glándula pancreática (Tabla 1). Las indicaciones más frecuentes para realizar un procedimiento de Puestow reportadas fueron: dolor en 21 pacientes $(46.7 \%)$, episodios de pancreatitis aguda a repetición en 17 (37.8\%) casos, tres $(6.7 \%)$ por conducto desconectado y dos (4.4\%) por insuficiencia pancreática. Dentro de los hallazgos transoperatorios se describió de consistencia dura al páncreas en 14 pacientes (31\%). El promedio del diámetro del conducto pancreático reportado fue de $8.19 \mathrm{~mm}$ y en $20(44.4 \%)$ pacientes se extrajeron litos del mismo. En 16 casos (35\%) se describió atrofia pancreática (Tabla 2).

El promedio de estancia hospitalaria fue de siete días, no se reportaron fístulas pancreáticas 
Tabla 1: Características generales de la población de estudio. $\mathrm{N}=45$.

\begin{tabular}{|c|c|}
\hline Variable & n (\%) \\
\hline Edad (años) & $46.44 \pm 14.59$ \\
\hline Sexo masculino & $24(53.3)$ \\
\hline Peso (kg) & $61.83 \pm 12.29$ \\
\hline Índice de masa corporal kg/m² & $22.71 \pm 4.11$ \\
\hline \multicolumn{2}{|l|}{ Antecedentes } \\
\hline Diabetes mellitus & $5(11.1)$ \\
\hline Consumo de alcohol & $19(42.2)$ \\
\hline Tabaquismo & $19(42.2)$ \\
\hline Índice tabáquico & $22.7 \pm 30.35$ \\
\hline Pancreatitis aguda & $37(82.2)$ \\
\hline Número de episodios de pancreatitis aguda & $5.2 \pm 5.38$ \\
\hline \multicolumn{2}{|l|}{ Insuficiencia pancreática } \\
\hline Endocrina & $14(31.1)$ \\
\hline Exocrina & $9(20.0)$ \\
\hline Diarrea & $19(40.0)$ \\
\hline \multicolumn{2}{|l|}{ Estudios de gabinete previos } \\
\hline Ultrasonido transendoscópico & $20(44.4)$ \\
\hline Resonancia magnética & $18(40.0)$ \\
\hline Tomografía axial computarizada & $44(97.8)$ \\
\hline \multicolumn{2}{|l|}{ Hallazgos preoperatorios } \\
\hline Calcificaciones & $32(71.1)$ \\
\hline \multicolumn{2}{|l|}{ Localización } \\
\hline Cabeza & $16(50.0)$ \\
\hline Cuello & $1(3.1)$ \\
\hline Cuerpo & $2(6.2)$ \\
\hline Proceso uncinado & $1(3.1)$ \\
\hline Múltiple & $12(37.5)$ \\
\hline \multicolumn{2}{|l|}{ Hallazgos preoperatorios } \\
\hline Litos & 15 (33.3) \\
\hline \multicolumn{2}{|l|}{ Localización } \\
\hline Cabeza & $11(73.0)$ \\
\hline Cuello & $1(7.0)$ \\
\hline Múltiple & $3(20.0)$ \\
\hline Atrofia & $14(31.0)$ \\
\hline Diámetro del conducto & $8.03 \pm 3.94$ \\
\hline \multicolumn{2}{|l|}{ Intervenciones previas } \\
\hline Endoscopia & 15 (33.3) \\
\hline \multicolumn{2}{|l|}{ Tipo de procedimiento } \\
\hline Litotripsia & $2(13.33)$ \\
\hline Stent pancreático & $4(26.66)$ \\
\hline Fallida & $2(13.33)$ \\
\hline Manejo del dolor & $1(6.66)$ \\
\hline Esfinterotomía & $3(20.00)$ \\
\hline Combinado & $3(20.00)$ \\
\hline Drenaje de pseudoquiste pancreático & $4(8.90)$ \\
\hline Necrosectomía pancreática & $2(4.40)$ \\
\hline Uso de enzimas pancreáticas & $23(51.10)$ \\
\hline Uso crónico de opioides & $4(8.90)$ \\
\hline
\end{tabular}

Los datos son presentados en media \pm desviación estándar o mediana (p25-p75). Variables categóricas se presentan en frecuencias y porcentajes.

Elaboración propia de acuerdo a resultados. y ningún paciente requirió manejo en terapia intensiva. A todos los pacientes $(100 \%)$ se les colocó algún tipo de drenaje durante la cirugía. Con respecto a las complicaciones, 14 (31\%) pacientes en total se caracterizaron según el sistema de clasificación de gravedad de las complicaciones quirúrgicas de ACCORDION: como leve en ocho (58\%) pacientes, moderada en tres $(21 \%)$ y grave en tres $(21 \%)$ (Tabla 2$)$.

En el seguimiento de los pacientes, 18 (40\%) cursaron con recurrencia del dolor; debido a esto, cinco (11\%) pacientes refirieron uso regular de opioides a pesar del procedimiento quirúrgico. Por otra parte, 20 (44\%) pacientes presentaron insuficiencia pancreática de novo, entre los cuales ocho (18\%) desarrollaron síntomas endocrinos como alteraciones de la glucosa sérica y 17 (38\%) presentaron insuficiencia exocrina manifestando diarrea o esteatorrea. Secundario a estos síntomas, 33 (73\%) requirieron uso de enzimas pancreáticas (Tabla 2). La tasa de reingreso hospitalario fue de 9\% (n $=4$ ). El seguimiento medio de los pacientes fue de 4.4 años.

En el análisis bivariado frente a la recurrencia del dolor, la edad mostró un valor de $\mathrm{p}$ de 0.274 , el sexo masculino $\mathrm{p}$ de $0.143 \mathrm{y}$ el peso de 0.439 (Tabla 3). En cuanto a los antecedentes, la relación frente a la historia de alcoholismo fue $p=0.139$ y a tabaquismo de $p=0.388$, al igual que en los que presentaban antecedentes de pancreatitis aguda y de procedimientos endoscópicos la p fue de 0.350 y 0.999 , respectivamente. El desarrollo de insuficiencia pancreática de novo posterior a la cirugía mostró una p de 0.67 frente al antecedente de pancreatitis aguda, mientras que de acuerdo a los hallazgos transoperatorios se evidenció en relación a la presencia de atrofia una $p=0.71$, consistencia dura del páncreas $p=0.90$, diámetro del conducto $p=0.47 y$ finalmente frente a la extracción de litos una $\mathrm{p}$ $=0.26$ (Tabla 4). No obstante, no se obtuvieron valores significativos en el análisis bivariado.

\section{DISCUSIÓN}

La pancreatitis crónica (PC) es una enfermedad compleja que implica una carga física, emocional y económica para el paciente, los médicos y el sistema de salud. En nuestra serie retrospec- 
Tabla 2: Características perioperatorias y de seguimiento.

n (\%)

Indicación de procedimiento de Puestow

Dolor

Conducto desconectado

$21(46.7)$

$3(6.7)$

$2(4.4)$

Insuficiencia pancreática

Episodios de pancreatitis aguda

Hallazgos transoperatorios

Consistencia dura del páncreas

Extracción de litos

Localización

Cabeza

Cuello

Cola

Múltiple

17 (37.8)

$14(31.1)$

$20(44.4)$

$9(45)$

$2(10)$

$2(10)$

7 (35)

Atrofia

16 (35)

Diámetro del conducto

Evolución postoperatoria

Inicio de dieta

$\geq 48$ horas

$12(27)$

$\geq 72$ horas

$18(40)$

4 a 7 días

$14(31)$

$>7$ días

Uso de drenaje

Fístulas pancreáticas

1 (2)

$45(100)$

$0(0)$

Días hospitalización postoperatorio

Complicaciones por ACCORDION

Grado

Leve

Moderada

Severa

Seguimiento

Recurrencia de dolor

Uso de opioides

Insuficiencia pancreática de novo

Endocrina

Exocrina

Uso de enzimas pancreáticas

Reingreso hospitalario

Seguimiento (años)

7 (5-9)

$14(31.1)$

8 (58)

$3(21)$

$3(21)$

$18(40)$

5 (11)

$20(44)$

8 (18)

$17(38)$

$33(73)$

4 (9)

$4.44 \pm 2.29$

Los datos son presentados en media \pm desviación estándar o mediana (p25-p75).

Variables categóricas se presentan en frecuencias y porcentajes.

Elaboración propia de acuerdo a resultados.

tiva se incluyeron 45 pacientes sometidos a un procedimiento de Puestow, como tratamiento quirúrgico descrito en PC. Esta revisión analizó los resultados obtenidos a lo largo de 19 años de experiencia, y a pesar de ser un análisis retros- pectivo, en México no hay descrita otra serie actual que analice la experiencia en relación a este tipo de procedimiento. Se han publicado diferentes técnicas quirúrgicas de drenaje, de resección o combinadas, ${ }^{23-25}$ sin embargo, el procedimiento de Puestow parece ofrecer menores tasas de morbilidad y mortalidad al no resecar tejido pancreático y preservar órganos funcionales como el duodeno.

El consumo de alcohol y tabaquismo se han identificado fuertemente como factores capaces de alterar la relación genética-ambiental de las personas, jugando un papel importante de riesgo en la evolución y desarrollo de la PC. Se sabe que las personas con antecedente de pancreatitis alcohólica tienen un riesgo de 12.5\% de desarrollar PC, pero hasta la fecha sólo en modelos animales se ha intentado comprender los mecanismos fisiopatológicos y genéticos que predisponen al desarrollo de PC, incluido el modelo SAPE descrito con anterioridad. ${ }^{8}$ Un hallazgo importante en nuestra serie fue que el $42.2 \%$ de los pacientes tenían antecedentes de tabaquismo y/o de consumo de alcohol; sin embargo, no fue posible relacionar directamente estos factores con el desarrollo de PC.

La elección del paciente para ser sometido a un procedimiento de Puestow como parte del tratamiento de PC debe ser en el contexto anatómico, tener un conducto pancreático dilatado (>5 mm), en ausencia de una masa inflamatoria de la cabeza del páncreas o de obstrucción del conducto biliar. ${ }^{11,18}$ Los hallazgos radiológicos patognomónicos de PC y los hallazgos transoperatorios de nuestra serie concuerdan con lo sugerido en otros estudios, ya que la media del diámetro del conducto pancreático entre ambos hallazgos fue de $8.11 \mathrm{~mm}$, sumado a la presencia de calcificaciones en la glándula y de litos en el conducto pancreático.

Otro aspecto importante a resaltar fue la ausencia de fístulas pancreáticas como complicación postoperatoria, y al analizar que la consistencia de la glándula era dura en todas las notas operatorias evaluadas, hace suponer que esta variable puede jugar un papel predictor importante en el desarrollo de fístulas pancreáticas, sin embargo, hacen falta estudios aleatorizados que puedan validar esta hipótesis.

De manera particular, los factores de riesgo para desarrollar fístula pancreática se han 
descrito con resultados significativos en pacientes sometidos a pancreatoduodenectomía; Zunxiang y colaboradores, basados en la definición del Grupo de Estudio Internacional de Cirugía Pancreática (ISGPS), describieron que 39.1\% de los pacientes con páncreas blando desarrollaron fístula grado $\mathrm{B}$ o $\mathrm{C}$, versus pacientes que tenían páncreas duro $(p<0.0001){ }^{26}$

Se han reportado tasas de éxito para el alivio del dolor a corto plazo después de un procedimiento de Puestow entre 80 a $85 \%$ de los pacientes y a largo plazo entre 70 a $80 \%$ durante cinco a 10 años de seguimiento. ${ }^{9,27}$ Sin embargo, se han descrito tasas mayores de recidiva del dolor (50\%) en aquellos pacientes con un diámetro del conducto pancreático $<7$ mm. $^{5}$

En una de las series más grandes incluidas en la revisión de Gouma DJ y su equipo, ${ }^{19}$ y descrita por Sakorafas GH y sus colegas, ${ }^{28}$ sobre los resultados obtenidos en la Clínica Mayo en el tratamiento quirúrgico de la PC, en 120 pacientes a los que se les realizó una pancreatoyeyunoanastomosis lateral, el grupo describió que la tasa de alivio del dolor fue de $81 \%$ con un seguimiento de hasta ocho años; en nuestros hallazgos, $60 \%$ cursaron sin recurrencia del dolor, con una media de reaparición del mismo de hasta 102 semanas y con un seguimiento promedio de 4.4 años después de la cirugía.

En el análisis bivariado de la recurrencia de este síntoma frente a variables demográficas como edad, sexo y peso, así también frente al antecedente de tabaquismo, alcoholismo y la realización de procedimientos endoscópicos previos a la cirugía, no se obtuvo una diferencia estadísticamente significativa que pueda comprobar la relación de estas variables como factores predictores de recidiva de dolor postquirúrgico luego de un procedimiento de Puestow; sin embargo, como se evidenció en nuestros resultados, $46.7 \%$ fueron llevados a cirugía bajo la indicación de dolor, seguido por el antecedente de pancreatitis aguda a repetición $(37.8 \%)$, por lo que esto supone que pueda existir sesgo en la descripción del dolor como indicación prequirúrgica, debido a la subjetividad de este síntoma que forma parte de las manifestaciones clínicas durante cualquier episodio de pancreatitis aguda.

El desarrollo de insuficiencia pancreática, manifestada por diabetes tipo 2 y/o diarrea/ esteatorrea de novo en el curso postoperatorio de un procedimiento descompresivo como el de Puestow, es menor comparado con lo re-

Tabla 3: Análisis bivariado de recurrencia del dolor postoperatorio.

\begin{tabular}{|c|c|c|c|}
\hline & $\begin{array}{l}\text { Recurrencia de } \\
\text { dolor }(\mathrm{N}=18)\end{array}$ & $\begin{array}{c}\text { Sin recurrencia } \\
\text { de dolor }(N=27)\end{array}$ & \\
\hline Parámetros & n (\%) & n (\%) & $\mathbf{p}$ \\
\hline Edad (años) & $49.39 \pm 11.96$ & $44.48 \pm 16.02$ & 0.274 \\
\hline Sexo masculino & $12(66.7)$ & $12(44.4)$ & 0.143 \\
\hline Peso (kg) & $63.59 \pm 10.63$ & $60.65 \pm 13.35$ & 0.439 \\
\hline Pancreatitis aguda previa & $16(88.9)$ & $21(77.8)$ & 0.350 \\
\hline Antecedente de tabaquismo & $9(50.0)$ & $10(37.0)$ & 0.388 \\
\hline Antecedente de alcoholismo & $10(55.6)$ & 9 (33.3) & 0.139 \\
\hline Antecedente de intervención por endoscopia & $6(33.3)$ & 9 (33.3) & 0.999 \\
\hline Páncreas atrófico* & 7 (38.9) & $8(29.6)$ & 0.600 \\
\hline Páncreas duro* & $6(33.3)$ & $8(29.6)$ & 0.999 \\
\hline Insuficiencia pancreática postquirúrgica & $11(61.1)$ & 9 (33.3) & 0.066 \\
\hline
\end{tabular}


Tabla 4: Análisis bivariado de insuficiencia pancreática de novo postoperatoria.

\begin{tabular}{|c|c|c|c|}
\hline & $\begin{array}{c}\text { Insuficiencia } \\
\text { pancreática de novo } \\
(\mathrm{N}=20)\end{array}$ & $\begin{array}{c}\text { Sin insuficiencia } \\
\text { pancreática de novo } \\
(\mathrm{N}=25)\end{array}$ & \\
\hline Parámetros & n (\%) & n (\%) & $\mathbf{p}$ \\
\hline Pancreatitis aguda previa & $17(85)$ & $20(80)$ & 0.67 \\
\hline Páncreas atrófico* & $6(30)$ & $9(36)$ & 0.71 \\
\hline Páncreas duro* & $8(40)$ & $6(24)$ & 0.90 \\
\hline Diámetro del conducto pancreático* & $3(3-10)$ & $4(3-12)$ & 0.47 \\
\hline Extracción de litos* & $7(35)$ & $13(52)$ & 0.26 \\
\hline
\end{tabular}

portado en los procedimientos de resección, y en relación a nuestros resultados se equiparó, aunque en menor tasa, con lo reportado en la serie de Sakorafas y su grupo ${ }^{28}$ (diabetes 18 vs $33 \%$ y esteatorrea 38 vs $40 \%$, respectivamente). No obstante, no hubo estadística significativa al querer comparar el desarrollo de insuficiencia pancreática de novo frente a los hallazgos transoperatorios del páncreas que pudieran predisponer al desarrollo de esta complicación.

\section{CONCLUSIONES}

Queda claro entonces, que dos de las metas en el tratamiento de la PC son el alivio del dolor y la mejora en la calidad de vida de los pacientes; si el tratamiento médico no consigue mitigar el dolor, la literatura actual sugiere que el tratamiento quirúrgico ha mostrado mejores resultados en comparación con tratamientos médico-endoscópicos. Este tipo de cirugías como el procedimiento de Puestow y otros que incluyen resección pancreática se llevan a cabo con baja morbilidad y mortalidad en centros de alto volumen como el Instituto Nacional de Nutrición y Ciencias Médicas "Salvador Zubirán".

En la actualidad, no existe un procedimiento quirúrgico ideal para pacientes con PC, y el tipo de intervención a elegir debe ser el reflejo del equilibrio entre el éxito del procedimiento y los riesgos inherentes. Aunque el alivio del dolor no se logró en $40 \%$ de los casos, dato que se equipara a lo reportado en otras series, debe considerarse el éxito alcanzado en el contexto del estado preoperatorio de los pacientes, mayormente en quienes ha fallado al tratamiento médico, radiológico y/o endoscópico, sumado al hecho de que el dolor crónico, incapacita y afecta la calidad de vida de las personas con diagnóstico de PC.

\section{REFERENCIAS}

1. Sarles H. Proposal adopted unanimously by the participants of the Symposium, Marseilles 1963. Bibl Gastroenterol. 1965; 7: 7-8.

2. Sarner M, Cotton PB. Classification of pancreatitis. Gut. 1984; 25: 756-759.

3. Whitcomb DC, Frulloni L, Garg P, Greer JB, Schneider A, Yadav D, et al. Chronic pancreatitis: An international draft consensus proposal for a new mechanistic definition. Pancreatology. 2016; 16: 218-224.

4. Conwell DL, Lee LS, Yadav D, Longnecker DS, Miller $\mathrm{FH}$, Mortele KJ, et al. American Pancreatic Association practice guidelines in chronic pancreatitis: evidencebased report on diagnostic guidelines. Pancreas. 2014; 43: 1143-1162.

5. Kleeff J, Stob C, Mayerle J, Stecher L, Maak M, Simon $\mathrm{P}$, et al. Evidence-based surgical treatments for chronic pancreatitis: a systematic review and meta-analysis of randomized controlled trials. Dtsch Arztebl Int Medicine. 2016; 113: 489-496.

6. Bausch D, Keck T. Pancreatic duct drainage procedure. In: Beger HG, Warshaw AL, Hruban RH, Buchler MW, Lerch MM, Neoptolemos JP, et al. The pancreas: an integrated textbook of basic science, medicine, and surgery, 3era ed. Oxford: Wiley; 2018, 453-457. 
7. Chang A, Abbott D. Chronic pancreatitis: puestow and frey procedures. In: Pawlik TM, Weber S, Gamblin TC. Case-based lessons in the management of complex hepato-pancreato-biliary surgery. USA: Springer; 2017, 385-399.

8. Yadav D, Whitcomb DC. The role of alcohol and smoking in pancreatitis. Nat Rev Gastroenterol Hepatol. 2010; 7: 131-145.

9. Bouwense SA, Kempeneers MA, Van Santvoort HC, Boermeester MA, van Goor H, Besselink MG, et al. Surgery in chronic pancreatitis: indication, timing and procedures. Visc Med. 2019; 35: 110-118.

10. Parekh D, Natarajan S. Surgical management of chronic pancreatitis. Indian J Surg. 2015; 77: 453-469.

11. Lohr JM, Dominguez-Munoz E, Rosendahl J, Besselink M, Mayerle J, Lerch MM, et al. United European Gastroenterology evidence-based guidelines for the diagnosis and therapy of chronic pancreatitis (HaPanEU) United European Gastroenterol J. 2017; 5: 153-199.

12. Jadad AR, Browman GP. The WHO analgesic ladder for cancer pain management. Stepping up the quality of its evaluation. JAMA. 1995; 274: 1870-1873.

13. Drewes AM, Kempeneers MA, Andersen DK Arendt-Nielsen L, Besselink MG, Boermeester $M A$, et al. Controversies on the endoscopic and surgical management of pain in patients with chronic pancreatitis: pros and cons! Gut. 2019; 68: 1343-1351.

14. Rosch T, Daniel S, Scholz M, Huibregtse K, Smits M, Schneider T, et al. European Society of Gastrointestinal Endoscopy Research Group. Endoscopic treatment of chronic pancreatitis: a multicenter study of 1,000 patients with long-term follow-up. Endoscopy. 2002; 34: 765-771.

15. Ke N, Jia D, Huang W, Nunes QM, Windsor JA, Liu X, et al. Earlier surgery improves outcomes from painful chronic pancreatitis. Medicine (Baltimore). 2018; 97: e0651.

16. Cahen DL, Gouma DJ, Nio Y, Delhaye M, Rauws EA, Boermeester MA, et al. Endoscopic versus surgical drainage of the pancreatic duct in chronic pancreatitis. N Engl J Med. 2007; 356 (7): 676-684.

17. Yang CJ, Bliss LA, Schapira EF, Freedman SD, Chau S, Windsor JA, et al. Systematic review of early surgery for chronic pancreatitis: impact on pain, pancreatic function, and re-intervention. J Gastrointest Surg. 2014; 18: 1863-1869.

18. Partington P, Rochelle R. Modified Puestow procedures for retrograde drainage of the pancreatic duct. Ann Surg. 1960; 152: 1037-1043.

19. Gouma DJ, Bornman PC. Surgery for chronic pancreatitis: pancreatic duct drainage procedures. In: Adams DB, Cotton PB, Zyromski NJ, Windsor JA. Pancreatitis: medical and surgical management, Holanda: Wiley; 2017, 273-278.

20. Dua M, Visser B. Surgical approaches to chronic pancreatitis: indications and techniques. Dig Dis Sci. 2017; 62: 1738-1744.
21. González M, Herrera MF, Laguna M, Gamino R, Uscanga L, Robles-Díaz G. Pain relief in chronic pancreatitis by pancreatico-jejunostomy. An institutional experience. Arch Med Res. 1996; 28: 387-390.

22. Dite P, Ruzicka M, Zboril V, Novotny I. A prospective, randomized trial comparing endoscopic and surgical therapy for chronic pancreatitis. Endoscopy. 2003; 35: 553-558.

23. Klempa I, Spatny M, Menzel J, Baca I, Nustede R, Stockmann F, et al. Pancreatic function and quality of life after resection of the head of the pancreas in chronic pancreatitis. A prospective, randomized comparative study after duodenum preserving resection of the head of the pancreas versus Whipple's operation. Chirurg. 1995; 66: 350-359.

24. Frey CF, Smith GJ. Description and rationale of a new operation for chronic pancreatitis. Pancreas. 1987; 2: 701-707.

25. Pericoli RM, Gourgiotis S, Alfieri S, Di Miceli D, Rotondi F, Quero G, et al. Indications and outcomes of surgical management of chronic pancreatitis: literature review. G Chir. 2007; 28: 164-174.

26. Ke Z, Cui J, Hu N, Yang Z, Chen H, Hu J, et al. Risk factors for postoperative pancreatic fistula: Analysis of 170 consecutive cases of pancreaticoduodenectomy based on the updated ISGPS classification and grading system. Medicine. 2018; 97 (35): 1-6.

27. Thuluvath PJ, Imperio D, Nair S, Cameron JL. Chronic pancreatitis: long-term pain relief with or without surgery, cancer risk, and mortality. J Clin Gastroenterol. 2003; 36: 159-165.

28. Sakorafas $\mathrm{GH}$, Farnell MB, Farley DR, Rowland CM, Sarr MG. Long-term results after surgery for chronic pancreatitis. Int J Pancreatic. 2000; 27: 131-142.

Consideraciones y responsabilidad ética: Privacidad de los datos. De acuerdo a los protocolos establecidos en nuestro centro de trabajo, se declara que se han seguido los protocolos sobre la privacidad de datos de pacientes y preservado su anonimato.

Financiamiento: No se recibió apoyo financiero para la elaboración de este trabajo.

Conflicto de intereses: Ninguno de los autores tiene conflicto de intereses en la realización de este estudio.

Correspondencia:

Dr. José Roberto Contreras-Ramírez

E-mail: dr.jrcontreras@gmail.com 